\title{
संगीत शिक्षण पद्धति एवं युगकालीन परिवर्तन
}

डा. सोनदीप मोंगा

एसिसटैंट प्रोफैसर, पंजाबी विभाग, सरकारी कालेज़, रोपड़

मानव चेतना एक अनुठी सच्चाई है। समस्त कायनात में यह विलक्षणता मनुष्य के पास ही है। मनुष्य के लिए सारा विश्व ही विद्या का विषय है बस इसका लक्ष्य-मार्गी होना अति आवश्यक है, जिसके लिए इसे सीखना पड़ता है, जिससे वह अपनी सांस्कृतिक पहचान बनाता है। इसका इतिहास, गौरव, आर्थिकता, जीवन शैली और उन्नति सब चेतन ज्ञान पर निर्भर है। इस लिए मानव विकास संस्कृति में ज्ञान सबसे उत्तम अभिधारणा है और ज्ञान प्रदान करने वाले गुरू का स्थान सबसे ऊँचा। भू-लोक को उजाले से भरने के लिए जो स्थान सूर्य का है, मानव जीवन को ज्ञान से आलोकित करने में वही स्थान गुरू का है। गुरू में सागर की गहराई, आकाश की ऊँचाई और धरती की विराटता है। गुरू ही विद्या दान देकर मानव जीवन को जीने योग्य लक्ष्य दृष्टि देता है। इस प्रकार मानव चेतना सही दिशा में अग्रसर रहती है और मानव कल्याण निश्चित हो जाता है।

\section{गुरू बिनु भवनिधि तरह न कोई जो बिरंचि संकर सम होई 11}

अर्थात कोई भी व्यक्ति गुरू के मार्गदर्शन के बिना अपने जीवन में सफल नहीं हो सकता, चाहे वह ब्रह्मा और महेश जैसे देवता के सम्मान क्यों न हो। भारतीय संस्कृति परम्परा में अध्यात्म, दर्शन, योग, चिकित्सा, साहित्य, कला तथा विज्ञान आदि जीवन के प्रत्येक क्षेत्र में गुरू की भूमिका अत्यन्त अपेक्षित और महान है। टी. एस. सोढ़ी लिखतें है:

'Teacher is considered to be most important factor to help the students in a spiritual way such a teacher is imbuild fully with his degree of self-knowledge, self-dynamism and spiritualism. He serves as a role- model. He guides the child with genuine love, afection and sympathy that he attains his full mental and spiritual development.'2

इतिहास की तरफ देखने से पता चलता है कि गुरू वशिष्ठ को पाकर श्री राम, अष्टावक को पाकर राजा जनक, गुरू संदीपनी को पाकर श्री कृष्ण, गुरू नानक को पाकर अंगद देव, बाबा हरिदास को पाकर तानसेन, स्वामी विरजानंद को 
पाकर दयानन्द, स्वामी रामकृष्ण परमहंस को पाकर विवेकानन्द, अलाऊद्दीन खाँ को पाकर रविशंकर धन्य हो गये, महान हो गये और स्वयं गुरू हो गये। इस प्रकार भारत में गुरू-शिष्य परम्परा सदीयों से चली आ रही है।

व्याकरण शासत्र की दृष्टि से गुरू शब्द की व्युत्पति दो शब्दों के योग से हुई है-गु+रू 'गु' का अर्थ है अंधकार और 'रू' का अर्थ है दूर करना। भाव जो अज्ञान रूपी अन्धकार को नष्ट कर ज्ञान रूपी, प्रकाश करता है वही गुरू है। हिन्दी विश्वकोष के अनुसार गुरू सम्प्रदाय प्रवर्त्तक, धर्मोपदेशक, किसी कला में निष्णात् व्यक्ति, शान्त-दान्त, कुलीन, विनीत, शुद्धवेषी, शुद्धाचारी, सुप्रतिष्ठित, पवित्र, दक्ष, आक्षम, व्यवस्था को मानने वाला ध्यान में लीन, मंत्र-तंत्र का ज्ञाता, निग्रह और अनुग्रह में समर्थ व्यक्ति।'3 संस्कृत हिन्दी कोष के अनुसार 'गुरू वह है जो सत्य वेद ज्ञान से जरा भी कष्ट न पहुँचाता हुआ अर्थात् जो विनम्र हो, मोक्ष प्राप्ति हेतु ज्ञान को देता हुआ दोनों कानों को खोल देता है और माता-पिता मे समान कभी द्रोह न करने योग्य होता है।'4 गुरूशवदरतनाकर-महानकोष के अनुसार गुरू अर्थात धर्म उपदेशक धर्म का आचार्य।5 मानक अंग्रेजी-हिन्दी कोष के अनुसार गुरू: शिक्षक, अध्यापक, मुदर्रिस, उपदेशक, पीर उस्ताद।'6 शब्दकोष की दृष्टि से तो शिक्षक और अध्यापक गुरू शब्द के ही समानार्थी है। गुरू के लिए कई पर्यायवाची शब्द भी जैसे मुरशद, उस्ताद, रहिबर, आचार्य आदि। गुरू शब्द से भाव उत्पन्न होते हैं कि गुरू दीपक के भांति प्रकाश देता है और केवल ज्ञान-दान ही नहीं देता अपितु शिष्य की चेतना को जागृत कर देते है।

शिष्य जो ज्ञान के प्रति जिज्ञासा रखता है, पाने की इच्छा रखता है, क्यों कि मनुष्य अपने आप में संपूर्ण नहीं हो सकता और उसकी कमीयां उसकी कमजोरी होती है। अपने आप को विकसित करने की इच्छा रखने वाला शिष्य है। लेकिन उसमें परमात्म शक्ति है जिसको दिशा मिलना आवश्यक है। यह शक्ति की लालसा ही उसे सीखने और पाने की और ले जाती है। डा. अमर सिंह धालीवाल मानव की इस रूचि को मनोवैज्ञानिक ढंग से ब्यान करते हुए लिखते हैं: 'मनुष्य एक सोचवान, याद रख सकने वाला और कल्पना कर सकने वाला जीव है। इस लिए वह कल्पनाओं से भ्रमित होता है उससे डरता है प्रभावित होता है। मनुष्य का व्यवहार वस्तुओं को जानने तक ही सीमित नहीं अपितु इसकी लालसाएं, कामनाएं भी होती है, उनके पुरे होने पर उनको खुशी होती है और असंतुष्टी से वह दुखी होता है।7 
शिष्य वह है जो अपने इस व्यवहार को साधना चाहता है और साधक भी कहलाता है। जैसे संतान माता-पिता की पहचान होते हैं वैसे ही शिष्य गुरू की पहचान होते है उसकी सफलता की कसौटी होता है। भारतीय गुरू-शिष्य परम्परा में गुरू शिष्य को अपने से उत्तम बनाने की कामना करता रहा है। लेकिन जैसे सदगुरू मिलना आवश्यक है वैसे गुरू को भी अच्छे विद्यार्थियों की तलाश होती है जैसे काची माटी को ढ़ालने पर सुन्दर मूर्त बनती है लेकिन माटी का सही होना भी अनीवार्य है वैसे ही शिष्य का धैर्यवान और आज्ञाकारी होना आवश्यक माना जाता रहा है।

वैदिक काल में हिन्दु सनातन धर्म के अनुसार तो गुरू को ही परमात्मा का स्थान दिया गया है और गुरू को ब्रह्मा विष्णु, महेश की उपाधि दी जाती थी। माता-पिता के स्थान पर भी गुरू को ही रखा जाता था। तब गुरू शिष्य परम्परा का प्रचलन धर्म स्वरूप था। धार्मिक मंत्र-उच्चारण से शरूति और स्मृति को जीवित रख कर पाठशाला की नींव रखी गई । जहां गुरू शिष्य को अभ्यास, मेहनत, लगन और सर्मपण का ज्ञान देकर आदर्श मनुष्य बनाता था। क्योंकि शुरू में कोई लिखित ग्रन्थ उपलब्ध नहीं थे, गुरू के अनुभव से ही ज्ञान परम्परा और गुरू शिष्य परिपाटी का आरम्भ हुआ। शिष्य अपने घर से मीलों दूर गुरूकुल में रहकर ज्ञान प्राप्ती किया करते थे और गुरू को ही माता पिता मानते। सुपरिचित श्लोक यहाँ द्रष्टव्य है:

\section{गुरूर्ब्रह्मा गुरूर्बिष्णु गुरूर्देवो महेश्वर}

गुरूर्साक्षत् परब्रहम, तर्में श्री गुरवे नमः । 18

तीन महान् शक्तियाँ ब्रह्मा, विष्णु और महेश मानी गयी है। उपरोक्त श्लोक में इन तीनों का समावेश गुरू को दिखाया गया है। भाव यह कि गुरू के पास वह सिद्धि है जो परमतत्व से व्यक्ति को एकाकार कर देती है, जिससे यह तीनों शक्तियां गुरू के द्वारा साधक के अंदर साकार हो जाती है। गुरूकुलों में गुरू और साधक वर्षों तक एक साथ रहते थे यह समय कई बार 15-20 वर्ष का भी होता था और वहीं पे जीवनचर्या का ज्ञान अभ्यास करते, क्योंकि गुरू-शिष्य के सम्बन्ध में भावानात्मक रिश्ते की जरूरत होती है उससे ही शिक्षण प्रकिया पूर्ण होती थी और दिल से साधक गुरू का सम्मान कर आज्ञाकारी बनता। रामायण में राम और हनुमान, महाभारत में कृष्ण और अर्जुन का सम्बन्ध इसकी उत्तम उदाहरण हैं परन्तु तब तक यह परम्परा मौखिक रूप में ही चलती थी। वेदों में ब्रह्मा का ज्ञान गुरू द्वारा शिष्य को मुखारबिन्दु से उच्चरित कर दिया गया था। उपनिषदों का ज्ञान 
आधार भी यही था अगर इसका शब्दिक अर्थ लें तो 'उप' भाव नजदीक, 'नी' भाव नीचे और 'षद' भाव बैठना अर्थात अध्यात्म गुरू के पास नीचे बैठ कर ज्ञान अर्जित करना।

भारत में जब अध्यात्म शिक्षा अभ्यास पद्धति में अपनाई गई तो भक्ति योग इसका स्वरूप बना। योग साधना में भी गुरू शिष्य परम्परा वैसे ही रही और गुरू को अनन्त और अखन्ड दर्जा दिया गया। कोई शास्त्र, कोई तपस्या, कोई मन्त्र, कोई दृश्य शैय, कोई जप गुरू के तुल्य नहीं है। केवल गुरू की साधना के द्वारा ही सिद्धि पाई जा सकती है, ऐसा लोगों का विश्वास रहा है। भक्ति योग और योग साधना मे तो बुद्धि से ज्यादा अनुभव की आवश्यकता होती है जिसके लिए एक निदेर्शक और पथ प्रदर्शक ही अनिवार्य है। भक्ति की शक्ति सीधे तौर पर या भैतिक तरीके से गुरू साधक को प्रदान नहीं कर सकता इसके लिए गुरू-शिष्य के सान्निध्य रिश्ते की आवश्यकता होती है। जब किसी साधक को शक्तिपथ अथवा दीक्षा दी जाती है तो सिद्धि ग्रहण होती है, तब सिद्धि प्राप्ति (गुरू-शिष्य सम्बन्ध) को मिटाती हुई उनका एकाकार कर देती है। मुन्डमंत्र में कहा जाता है कि सिद्धि का मूल देवता में होता है, देवता का मूल मंत्र है, मंत्र का मूल दीक्षा में है और दीक्षा का मूल गुरू है। जैसे देवता को अराधना के द्वारा पाया जा सकता है वैसे गुरू की सेवा और पूजा से ज्ञान पाया जा सकता है यहां जक माना जाता है कि सभी तीर्थ स्थानों का जप तप गुरू-चरणों मे बसता है। भक्ति योग में इसे शिष्य के द्वारा गुरू को सम्पूर्ण सर्मपण की अभिधारणा के तौर पे माना जाता हैं गुरू शिष्य परम्परा की यही परिपाटी आरम्भ काल से ही प्रचलित है कि अपने अहम को पूर्णतय त्याग करना ही पाना है और गुरू के आगे समर्पण भाव से ही शिष्य पा सकता है। शिष्य की यही निर्भता उसे गुरू को माता-पिता के तुल्य मानने पर सजज् करती है।

थेरवादी बोध परम्परा में भी गुरू शिष्य परम्परा का प्रचलन रहा है। इस परम्परा के अनुसार गुरू को प्रकाश प्रदान करने वाला माना गया है और शिष्य को सम्मान देने वाला गुरू अपने शिष्य के हृदय में सूर्य के भांति रात-दिन सदा प्रकाश फैलाता रहता है, वह शिष्य के अज्ञान रूपी अंधकार का नाश कर देता है उसकी सारी व्याधियां नष्ट करके उसको शांति स्वरूप बनाता है। इसके अन्त्रगत गुरू के आचरण अनुसार व्यवहार करने का आहवान किया गया और महात्मा बुद्ध को गुरू दीक्षा देने के कारण भगवान माना गया। शान्तिदेव बुद्ध ने बोधीसत्व पाने वाले 
भिक्षुको को मानव कल्याण हेतू तैयार किया बोधीसत्व के मार्ग पर चलने वाले साधकों को वोधिचित का विकास करना पड़ता था। बोधीचित की प्रार्थनाओं से यह स्पष्ट हो जाता है कि वोधीसत्व का चरित्र निस्वार्थ समाज सेवक का ही चरित्र था, उनकी प्रार्थनाएं प्रतिज्ञा और संकल्प हुआ करती थी।'9

सिक्ख गुरू साहिबान तथा अन्य सन्त-भक्तों की वाणी के महान संग्रह 'गुरू ग्रंथ साहिब' में गुरू महिमा से सम्बन्धित अनेक पद संकलित हैं स्वंय गुरू ग्रंथ साहिब को सिक्खों ने ज्ञान स्रोत होने के कारण जीवंत गुरू का दर्जा दिया। सच्चाई का ज्ञान करवाने वाले गुरू नानक देव जी गुरू की महिमा इस प्रकार गाते है:

\section{कुंभे बधा जल रहै जल बिनु कुंभु न होई}

गिआन का बधा मनु रहै गुर बिनु गिआन न होई 110

अर्थात जैसे जल को घड़े में ही संचित करके रखा जा सकता है बिना पात्र के जल को बांध कर रखना सम्भव नहीं है। इसी प्रकार मन का नियंत्रण ज्ञान से ही हो सकता है और ज्ञान गुरू के बिना प्राप्त नहीं हो सकता। भाव यह है कि जीवन को सुचारू ढ़ंग से संचालित करने के लिए ज्ञान प्राप्ति के लिये सदगुरू का सान्निध्य आवश्यक है। ऐसे ही गुरू अंगद देव जी जिन्होने गुरू नानक देव जी का गुरू के तौर पे सान्निध्य प्राप्त किया गुरू की महिमा का सुन्दर चित्रण करते हुए श्लोक प्रस्तुत करते हैं:

\section{जे सउ चंदा उगवहि, सूरज चड़हि हजार \\ ऐते चानण होंदिआ गुरू बिन घोर अंधार । 11}

अर्थात सूर्य चाँद तो शास्त्रों के प्रतीक मात्र हैं। कोई भी व्यक्ति यदि शास्त्रों का अध्ययन करके ज्ञानवान् तो हो सकता है परन्तु विवेक बुद्धि गुरू के सान्निध्य में गूढ़ तथ्यों को आत्मसात् करके ही प्राप्त की जा सकती है। सौ चन्द्रमा उदय हो जाएं हजार सूर्य प्रकाशमान हो जायें, इन सबके रहते गुरू बिना जीवन में अंधकार ही रहता है भावार्थ यह है कि सूर्य चन्द्रमा तो बह्म जगत को प्रकाशित करते हैं आंतरिक ज्ञान तो गुरूदेव के कमल स्वरूप चरणों की वंदना से ही प्राप्त हो सकता है।

गुरू नानक देव जी ने 'वाहेगुरू' गुरूमंत्र देकर मनुष्य को यह बोध करवाने का प्रयन्त किया कि गुरू की सर्वदा जय है वह परमचेतना सब गरूओं का भी गुरू है 
जिसे हमें अपने भीतर प्रवाहमान रखता है। उन्होने अपने मंत्र को अपनाने वालों को 'सिक्ख' की संज्ञा दी, जिसका अर्थ है सर्वदा सीखने वाला। यह गुरू -शिष्य परम्परा की नई परिपाटी सिद्ध हुई। गुरू गोबिन्द जी ने इसे एक अनुठी 'गुरू-चेला' पहचान दी और यहां तक के अपने आप को भी शिष्य ही ब्यान किया। जिससे सभी साधक जीवन में सही मार्ग पर चलते हुए अपेक्षित शुभ कर्म करें। गुरू तत्व से जुड़ने के लिए उन्होने साधको की संगत पर भी बल दिया। कबीर जी ने तो गुरू को गोविंन्द से भी उच्चाँ स्थान दिया।

सभी मत्तों के विश्लेषण से ज्ञात होता है कि प्राचीन भारतीय परम्परा और मानव विकास संस्कृति में शिक्षण-प्रशिक्षण का पज्ञ गुरू शिष्य प्रणाली के द्वारा सम्पन्न किया जाता रहा है और इतिहास के अनुसार यह प्रणाली सभी ज्ञान विधाओं पर लागु होती रही है जैसे कि धर्म, साहित्य, कला,दर्शन, राजनीति-शास्त्र तथा ओषधि-विज्ञान आदि।

अगर बात संगीत शिक्षा की करें तो वैदिक युग से ही संगीत शिक्षा में गुरू शिष्य परम्परा का प्रचलन रहा है। गुरूकुल शिक्षा को संगीत के लिए भी प्रयोग किया जाता रहा है कि जब कोई गायक, वादक एवंव नर्तक श्रेष्ठ गुरू के संरक्षण में रहकर निरन्तर संगीत साधना करता और साथ-साथ आचार-विचार, रीति, नियम आदिक की अनुशासनात्मक शिक्षा भी दी जाती थी। यह भी प्रचलन था कि उन शिष्यों में सर्वश्रेठ शिष्य को गुरू के पास उस आश्रम में ऋषियों द्वारा संगीत की प्रयोगिक शिक्षा देने का भी उल्लेख हैं प्राचीन और मध्यकाल में भी गुरू शिष्य परम्परा संगीत का विकास मत, परम्परा, सम्प्रदाय आदि के रूप में होता रहा है। वंश परम्परा भी गुरू-शिष्य संगीत परम्परा बनी। क्यों राज दरबारों में गाने-बजाने वाले कलाकार अपनी औलाद को ही यह संगीतक कला कौशल सिखाते, ता कि उनकी जीविका चलती रहे इस तरह पिता-पुत्र भी गुरू शिष्य के तौर पर निर्वाह करते थे। लेकिन गुरू की भूमिका इसमें सक्रिय रहती थी। जैसे कि माना गया है:

'Our music tradition has survived through 'Guru Shishya' parampara, Guru spends several hours imparting training and teaching the intricacies of the art form to the shishya, which a shishya with his hard parctice and sadhana polishes up to present the most sublime art form. Thus the relation between Guru-Shishaya has been given the utmost importance in Indian music. Even the son-father relation becomes secondary in this art form.'12 
कालाअन्तर के साथ संगीत शिक्षा में घरानेदार कलाकारों का महत्त्वपूर्ण स्थान बना किसी विशेष राग एवं ताल के स्वरूपों को व्यवहार करते हुए यह शिष्य कलाकार अपने गुरूओं की शैली की मान्यताओं के अनुसार परिपक्वता प्राप्त करते। जिससे एक नवीन संगीतक कौशल का निर्माण होता है। गायन वादन एवं नृत्य की इस परम्परागत पद्धति को गुरू शिष्य परम्परा में 'घराना' नाम दिया गया। जिसमें हरेक घराना किसी तालों, रागों के स्वर प्रयोग करने की विशेष विधि के साथ सामने आता गया। इस परम्परा में ग्वालियर, आगरा, दिल्ली, जयपुर, किनाना, पटिआला, बनारस, मैहर, रीवा घराने विकसित हुए। इनमें किसी घराने के गुरू एवं शिष्य उस घराने की विशेष शैली में अभ्यास करते हुए गुरू एवं घराने का नाम ऊँचा रखने का प्रयास करते हैं।

प्राचीन काल से अब तक की संगीत शिक्षा पद्धति में गुरू और शिष्य के बीच पवित्र पारस्परिक सम्बन्ध का अंश था। उस्ताद-शार्गिद का यह बँधन स्वार्थ से रहत होता। यहां शिष्य संगीत के साथ-साथ दर्शन, धर्म, ध्यान साधना को भी ग्रहण करने के सुपात्र बनते । इस तरह भी कहा जा सकता है कि इन समयों के दौरान संगीत पूर्ण रूप से गुरू-प्रधान विद्या रही, जिसमें एक कुशल गुरू अपने समयक ज्ञान, कड़े अभ्यास एवं योग्य मार्गदर्शन के द्वारा अमुल्यवान संगीतक धरोहर को शिष्यों में बांटते रहे जिससे संगीत की निर्मल धारा प्रवाहित रही।

वर्तमान समय में संगीत शिक्षण का कार्यरूप संस्थागत हो गया है। भारतीय शास्त्रीय संगीत पद्धति में संस्थागत शिक्षण प्रणाली का प्रभाव सबसे पहले पड़ा। संगीत नित्य नूतन हैं। नाट्य संगीत चित्रपट संगीत, भक्ति संगीत, एवम लोक संगीत और गीत-गजल को अधिक सीखने की इच्छा ने संगीत की उस्ताद-शागिर्द परम्परा को जीवति रखा। इसी लिऐ संगीत कला को ऐच्छिक विषय के तौर पर संस्थागत रूप में विद्यायलयों, महाविद्यायलयों और विश्वविद्यायलयों में मान्यता मिलनी आरम्भ हुई। शुरू में यह संगीत शिक्षण गुरू-शिष्य प्रणाली पर ही आधारित रहा क्यों कि यहाँ वह लोग आए जो गुरू शिष्य प्रणाली के अधीन ही प्रशिक्षित थे। लेकिन इसका स्वरूप आधुनिक हो गया जैसे विद्यार्थी की अभिरूचि तथा क्षमता का अन्वेषण, व्यक्तिनिष्ठ संगीत-शिक्षण नियमित पठन-पाठन को प्रोत्साहन, मंच-प्रदर्शन हेतु प्रेरणा नियमित अभ्यास, मनोवैज्ञानिक तकनीकी तत्वों का आधार प्रदान करना हो गया। यही आधार बिन्दु संगीत को 'विशेष' और 'उच्चतर' श्रेणी तक ले गये। 
इसके चार मुख्य, अंग सामने आए: शिक्षक, शिक्षार्थी, शिक्षण पद्धति एवं मूल्यांकन। लेकिन संस्थागत संगीत के अमल में एक कमी रह गई कि इसको प्राथमिक एवं माध्यमिक स्तर पर महत्व नहीं दिया गया कि इसको प्राथमिक एवं माध्यमिक स्तर पर महत्व नहीं दिया गया और न ही आवश्यक पाठयक्रम का भाग बनाया गया बल्कि इसे एक ऐच्छिक विषय के तौर पर उच्चतर स्तर पर अपनाया गया जिससे गुरू शिष्य परम्परा का वजूद हिलने लगा क्यों कि अब गुरूजन और शिष्य एक साथ रियाज करने के लिए समय सीमा में बंधे हैं। उनको निश्चित समय में निश्चित पाठयक्रम पूरा करना होता है। समर्पण, धैर्य, लम्बा अभ्यास और गुरू शिष्य परिपाटी इसमें गायब है, क्यों कि इस भौतिकवादी प्रौद्यौगिक युग में जीवन इतना गतीशील हो गया है कि कला साधना को शिष्य सम्पूर्ण समपर्ण नहीं देते और गुरू से भी लगाव कम हो गया है। व्यवसायिकरण की दौड़ के कारन संस्थागत संगीत शिक्षण 'जाब ओरियेन्टेड़' बनने लगा है। ग्लोबलाइजेशन के इस युग में यह परिवर्तन स्वभाविक है। लेकिन यह कोलाहल संगीत पद्धति और गुरू-शिष्य परम्परा को नष्ट न कर दे यह डर भी बना हुआ है। संगीत कला का लक्ष्य आनन्द प्राप्ति ही नहीं समाज को ज्ञान साधना से जोड़ना भी है लेकिन संगीत में मीड़िया व ग्लैमर संगीत को मुल्यविहीन कर रही है।

प्रो स्वतन्त्र शर्मा जी इस समस्या का गहन अध्यन करते हुए लिखते हैं विद्यालयों एवं विश्वविद्यालयों में संगीत शिक्षण प्रारम्भ होने से जहाँ एक और संगीत सीखना सर्वसुलभ हो गया वहीं संगीत शिक्षण से सम्बन्धित कई समस्याएं भी उठ खड़ी हुई। यद्यपि, संगीत आज सर्वसुलभ है किन्तु संगीत के आन्तरिक तत्त्वों को उभरने का अवसर नहीं मिल पा रहा है।'13 प्राचीन गुरू शिष्य परम्परा में संगीत शिक्षण मौखिक ढ़ंग से दिया जाता है गुरू शिष्य को ज्ञान कंट करवाता था। संस्थागत संगीत में शिष्यों के पास सीखने के लिए सुलभ साधन भी मौजुद है जैसे टेप-रिकार्डर, वॉइस रिकार्डर, सी.डी. वीडियो कॉलिंग-श्रव्य-दृश्य पुस्तकालय, इन्टरनैट। वह जब चाहे इससे पुनः स्मरण कर सकते हैं इससे शिष्य के मन में गुरू की और सम्पूर्ण नहीं हो पाता जिससे वह संगीत शिक्षण तो पा लेता है, लेकिन उसके साथ जुड़ी मुलय-साधना से दुर हो जाता है। परन्तु यह भी नहीं कहा जा सकता कि संगीत शिक्षण को संस्थागत प्रबन्धन की आवश्यकता नहीं है। यहां पर डॉ. तृप्त जी का मत सही है कि एक युग की मांग गुरू-शिष्य परम्परा थी तो आज 
के युग और परिस्थितियों की मांग दुसरी संस्थागत शिक्षण प्रणाली है। परन्तु इसका अर्थ यह भी नहीं कि दोनो में से किसी एक को अधिक उपयोगी और दूसरी प्रणाली को कम उपयोगी कहा जा सकता है। वास्तविकता यह है कि इन दोनों व्यवस्थाओं की अपनी-अपनी विशेषताएँ और कमियां हैं गुरू-शिष्य पद्धति का प्रभाव पैना और गहरा है तो संस्थागत व्यवस्था का प्रभाव व्यापक है। इसी प्रकार पहली पद्धति श्रम और समय की दृष्टि से लम्बी है तो दुसरी खर्चीली और सतही है। ऐसी स्थिति में आवश्यकता इस बात की है कि उपलब्ध दोनों व्यवस्थाओं में समय के साथ-साथ जो कमियां आ गई है। उन्हें दुर कर पुन: नईशक्ति और ऊर्जा के साथ क्रियान्वित किया जाए तो निश्चित रूप से अच्छे परिणाम सामने आयेंगे।'14 गुणवत्त्ता प्रयोग की दृष्टि से गुरू-शिष्य का रिश्ता अटूट है चाहे कोई भी युग हो और साधन कितने भी सुलभ क्यों न हो। संस्थागत सुलभता के साथ अगर गुरू-शिष्य पद्धति और संगीत मुल्य शिक्षण का समाजस्य हो तो न गुरू का महत्व कम होगा और न छात्र/शिष्य की योग्यता में कोई कमी। इस बंधन की विश्व व्यापक्ता पर किसी विद्ववान की यह पंक्तियाँ सही बैठती है:

We come from darkness

yet another darkness is ignorance

let the light be revealed to both teacher

and student there is relationship

let there be no hatred between two

let there be peace

let there be no disturbance

in the three structure of the body, the mind and senses

so that this commitment continues

so that this relationship flowers.

\section{सहायक स्रोत}

1. गोस्वामी तुलसीदास, रामचरितमानस, उत्त्तरकाण्ड $92 / 3$ पृष्ठ 921

2 T.S. Sodhi, G.S. Sandhu, S. Balwant Singh, Philosphies of Education, The Indian Publishers, Ambala, 1988, P. 185.

3. रामप्रसाद, त्रिपाठी, हिन्दी विश्वकोष, पृष्ठ 467

4. छज्जुराम शास्त्री, भगीरथ शास्त्री, देव शर्मा शास्त्री, संस्कृत-हिन्दी कोष, पृष्ठ 72-73

5. भाई काहन सिंह नाभा, गुरूशबदरतनाकर महानकोष, भाषा विभाग पंजाब, पटिआला, पुष्ठ 314 
6. सत्य प्रकाश और बलभद्र प्रसाद मिश्र (संम्पा:), मानक अंग्रेजी-हिन्दी कोष, पृष्ठ 389

7. डॉ. अमर सिंह धालीवाल, विद्यियक मनोविज्ञान, पब्लीकेशन ब्यौरो पंजाबी युनीवरसिटी पटिआला, 1977, पृष्ठ 4

8. गुरू गीता, सदगुरू की महिमा, पृष्ठ $17 / 1$

9. रामधारी सिंह दिनकर, सभ्याचार के चार अध्याय, पब्लीकेशन ब्यौरो पंजाबी युनीवरसिटी पटिआला 1992, पृष्ठ 208

10. आदि ग्रंथ, पृष्ठ 469

11. आदि ग्रंथ, पृष्ठ 462

12 Pandit Debu chaudhari, Indian Music and Ustad Mustaq Ali Khan, Preface.

13. प्रो. स्वतन्त्र शर्मा, सौन्दर्य रस एवं संगीत, पृष्ठ 288

14. डॉ. तृप्त कपूर, उत्तर भारत में संगीत शिक्षा, पृष्ठ 159 